\title{
Influencia del ejercicio físico en el desarrollo de fístulas arteriovenosas nativas
}

\author{
María Teresa López Alonsoํ, Valentín Lozano Moledoํㅜ, Anna Yuguero Ortiz², Néstor Fontseré Baldellou³ \\ ${ }^{1}$ Diplomada/o en Enfermería, ${ }^{2}$ Diplomada en Fisioterapia, ${ }^{3}$ Especialista en Nefrología. Unidad Funcional de Acceso \\ Vascular (UFAV). Sección de Diálisis. Servicio de Nefrología y Trasplante Renal. Hospital Clínic. Barcelona. España
}

\section{Resumen}

Objetivo: Evaluar la influencia de enfermería nefrológica de rol avanzado en un programa controlado de ejercicio físico post-operatorio sobre la maduración de la fistula arteriovenosa nativa (FAVn) al mes de su implantación.

Material y Métodos: Estudio prospectivo randomizado. Pacientes pre-diálisis y hemodiálisis. Criterios exclusión: discapacidad psíquica o física para realizar el programa de ejercicio. Tras la cirugía randomización a grupo ejercicio y control. Evaluación de criterios de maduración clínica y ecográfica. Análisis estadístico descriptivo, $\mathrm{X}^{2}$ y T- Student.

Resultados: 69 pacientes (48 varones), 66.8 (13.8) años y $65.2 \%$ en etapa de pre-diálisis.

38 grupo ejercicio y 31 control. Al mes se observaron tasas generales de adecuada maduración clínica y ecográfica del $88.4 \%$ y $78.3 \%$, respectivamente (Índice Kappa $=0.539$ ). Tan solo se observó un caso de disconcordancia entre ambos métodos. No se observaron diferencias significativas en los criterios de maduración clínica y ecográfica entre ambos grupos de estudio. En el análisis de regresión logística se detectó la localización como principal factor de confusión, revelando una significativa mayor maduración clínica pero no ecográfica en el subgrupo de pacientes portadores de FAVn distales $(94.7 \%$ vs $60 \%$; $P=0.019$ ).

\section{Correspondencia: \\ $\mathrm{M}^{\mathrm{a}}$ Teresa López Alonso}

Servicio de Nefrología y Trasplante Renal Sección de Diálisis. Hospital Clínic Barcelona C/ Villarroel n² 170. E. 08036 Barcelona E-mail:mtlopez@clinic.ub.es.
Conclusiones: 1.- Un programa de ejercicio controlado post-quirúrgico favorece una adecuada maduración clínica, especialmente en el subgrupo de pacientes con FAVn distales. 2.- Tan solo se observó un caso de disconcordancia entre los criterios de maduración clínica y ecográfica. 3.- De acuerdo a nuestros resultados recomendamos la realización de una pauta controlada de ejercicio físico, bajo la supervisión de una enfermera experta en hemodiálisis.

\section{PALABRAS CLAVE}

- INSUFICIENCIA RENAL CRÓNICA ESTADIO 5 (PRE-DIÁLISIS) Y 5D (HEMODIÁLISIS)

- ACCESO VASCULAR

- FÍSTULA ARTERIOVENOSA NATIVA

- EJERCICIO FÍSICO

Influence of physical exercise on the development of native arteriovenous fistulas

\begin{abstract}
Objective: The aim of this study was to determine whether a postoperative ambulatory controlled exercise program can increase arteriovenous fistulas (AVF) maturation at 1 month.

Material and Methods: In this randomized study we included a subgroup of pre- dialysis and hemodialysis patients. After surgery, all patients were randomized to an exercise group or a control group. At 1-month postoperatively, clinical maturation (hemodialysis nurse) and ultrasonographic maturation (nephrology) was assessed in all patients. Descriptive statistics, $\mathrm{X}^{2}$ test and Student T-test were use for statistically study.
\end{abstract}


Results: 69 patients (48 men), 66.8 (13.8) years old and $65.2 \%$ in pre-dialysis status. After randomization we study 38 patients in exercise and 31 in control group, respectively. At 1-month after surgery, clinical and ultrasonographic maturation was assessed in $88.4 \%$ and $78.3 \%$ of AVF (Kappa $=0.539$ ). Only one AVF deemed mature by ultrasound examination was considered immature by clinical examination. AVF localization was detected as a confounding factor for both clinical and ultrasonographic maturation, leading to a significant greater effect of exercise in maturation of distal AVF for clinical criteria ( $94.7 \%$ vs $60.0 \% ; P=0.019$ ).

Conclusions: 1.- A postoperative controlled exercise program after AVF creation seems to increase 1-month clinical AVF maturation, specially in distal access. 2.- We only observed one AVF deemed mature by ultrasound examination was considered immature by clinical examination. 3.- According to our results we can recommend physical exercise on the development of native arteriovenous fistula under control by hemodialysis nurse.

\section{KEYWORDS}

- CHRONIC KIDNEY DISEASE STAGE 5 (PRE-DIALYSIS) AND 5D (HEMODIALYSIS)

- VASCULAR ACCESS

- NATIVE ARTERIOVENOUS FISTULA

- PHYSICAL EXERCISE

\section{Introducción}

El principal objetivo de nuestra unidad de acceso vascular es establecer un conjunto actuaciones protocolizadas encaminadas a una atención integral y multidisciplinaria del acceso vascular (AV). Una de nuestras principales premisas es la implantación de una fístula arteriovenosa nativa $(F A V n)$ normofuncionante así como el tratamiento precoz de todas las complicaciones derivadas. Para ello resulta imperativo tener una consulta externa específica de acceso vascular bajo la supervisión de un equipo de coordinación integrado por enfermera de hemodiálisis y nefrólogo, que deberán establecer una comunicación constante y fluida con los equipos de cirugía vascular y radiología vascular intervencionista.

Diferentes estudios han evidenciado como los pacientes en hemodiálisis portadores de una FAVn presen- tan una mejor dosis de hemodiálisis, mayor tiempo de permeabilidad y menor número de complicaciones asociadas $^{1-3}$. Por lo contrario aquellos pacientes portadores de catéteres venosos centrales muestran peores curvas de supervivencia, secundarias a una mayor morbi-mortalidad derivada de las complicaciones infecciosas $^{4-5}$.

Las actuales guías de práctica clínica de acceso vascular $^{7-9}$, recomiendan la realización de ejercicio físico en el acceso con el principal objetivo de favorecer su desarrollo. A pesar de ello existen pocos trabajos al respecto con escaso número de pacientes ${ }^{10-12}$. El principal objetivo de nuestro estudio fue evaluar el efecto de un programa controlado de ejercicio físico post-operatorio, sobre la maduración de FAVn al mes de su implantación bajo la supervisión de enfermería de rol avanzado.

\section{Material y método}

Se trata de un estudio randomizado y prospectivo realizado de Junio de 2013 a Noviembre de 2014. Se incluyeron pacientes con insuficiencia renal crónica en etapa de pre-diálisis (estadio 5) y hemodiálisis (estadio 5D) derivados a nuestro centro para la implantación de una FAVn. Los criterios de exclusión fueron la discapacidad psíquica o física para entender o realizar el programa de ejercicio.

La información, evaluación e inclusión de los sujetos se realizó en la consulta externa de acceso vascular. En caso afirmativo de participación se firmó un consentimiento informado auditado previamente por el Comité de Ensayos Clínicos de nuestro centro. Antes de la implantación quirúrgica del acceso vascular, todos los pacientes realizaron ejercicios mediante una pelota de consistencia suave. Tras la implantación de la FAVn los pacientes fueron randomizados al grupo de ejercicio físico y control. Al grupo estudio se les entregó un programa protocolizado (tríptico) de diferentes ejercicios ambulatorios mediante la utilización de bandas elásticas (Figura 1). A los integrantes del grupo control se les aconsejó continuar con el estilo de vida habitual. El programa de ejercicio físico consistió en una tabla protocolizada con tres tipos diferentes de ejercicios diarios a realizar a partir de la primera semana tras la intervención: flexión- extensión del codo y muñeca, cierre y apertura de la mano mediante una banda elástica. En la Tabla 1 se especifica de una forma más detallada cada uno de los ejercicios y la frecuencia diaria. 
A todos los pacientes incluidos en el estudio se les midió la fuerza muscular mediante un Hand-Dynamometer (Seahan $®)$ y se les realizó una valoración clínica (enfermera de hemodiálisis) y ecográfica (nefrólogo), al mes de la implantación de la FAVn. Entre los criterios de una adecuada maduración clínica realizada por la enfermera se consideró una vena fácilmente palpable, una longitud $>10 \mathrm{cms}$ y la presencia de buen thrill. Los parámetros ecográficos se establecieron según las recomendaciones actuales de las guías europeas de acceso vascular (flujo $>500 \mathrm{~mL} / \mathrm{min}$, diámetro venoso $>5 \mathrm{~mm}$ y profundidad $<6 \mathrm{~mm})^{13}$.

La recogida de los datos se realizó mediante una ficha de estudio y el análisis estadístico mediante el programa estadístico SPSS versión 15 . Se realizó estadística descriptiva y se utilizaron los test de Chi-cuadrado $\left(\mathrm{X}^{2}\right)$ para las proporciones y T-Student para el análisis de las variables continuas. Se consideró significativa una $\mathrm{P}<0.05$.

\section{Resultados}

Se incluyeron un total de 69 pacientes ( $70 \%$ varones) con una edad media de 66.8 años (13.8 años). En la Tabla 2 se especifican con mayor detalle las características generales del grupo de estudio. Tras el procedimiento quirúrgico se implantaron un $42 \%$ de FAVn distales (FAV radio-cefálicas) y un $58 \%$ proximales (FAV húmero-cefálicas o húmero-basílicas con superficialización y trasposición). El grupo de estudio fue randomizado tras el procedimiento quirúrgico incluyéndose 38 pacientes en el grupo ejercicio y 31 en el control.

Al mes tras la implantación se observaron unas tasas generales de adecuada maduración clínica y ecográfica del $88.4 \%$ y $78.3 \%$ de las $F A V n$, respectivamente. Para el análisis de concordancia entre la valoración clínica (enfermera de hemodiálisis) y ecográfica (nefrólogo) se calculó el índice de Kappa que resultó de 0.539 (nivel medio-alto). Tan sólo se detectó un caso de disconcordancia entre los dos métodos de evaluación (una FAVn madura por criterios ecográficos e inmadura por exploración clínica). Los pacientes del grupo ejercicio presentaron un incremento significativo de la fuerza medida por Hand-Dynamometer respecto al control $(+1.65 \mathrm{Kg}$ [DT 2.81$]$ vs $-0.87 \mathrm{Kg}$ [DT 1.84], $P<0.001)$.

En el análisis estadístico de aquellos factores con mayor peso en la maduración de las FAVn se determinó la localización (distal o proximal) como uno de los principales factores de confusión. En la Figura 2 se muestran los porcentajes de maduración por criterios clínicos y ecográficos obtenidos en las FAVn distales y proximales. Según los resultados obtenidos aquellos pacientes del grupo ejercicio portadores de FAV radio- cefálicas (distales) presentaron una significativa mayor maduración clínica pero no ecográfica $(94.7 \%$ vs $60 \% ; P=0.019$ ).

\section{Discusión}

Según los resultados obtenidos en nuestro estudio recomendamos la realización de una pauta protocolizada de ejercicios post-operatorios para favorecer la maduración de FAVn, en especial en las de localización distal.

Son muy pocos los estudios que presentan datos sobre la eficacia del ejercicio para mejorar la maduración 0 supervivencia de la FAVn. Existen tres guías de práctica clínica que evalúan los ejercicios de dilatación en el acceso vascular ${ }^{7-9}$. Entre los posibles efectos beneficiosos reportados se incluye un aumento de la masa muscular y del diámetro venoso, así como una disminución en la cantidad de tejido adiposo. Sin embargo, las únicas que recomiendan su uso son las $\mathrm{K} / \mathrm{DOQI}{ }^{9}$. A pesar de ello, la mayoría de los estudios son no randomizados, con escaso número de pacientes y reportan los posibles efectos beneficiosos antes de la creación quirúrgica de la $\mathrm{FAVn}^{10-12}$.

Según los datos reportados en diferentes estudios entre un 50 y $70 \%$ de los pacientes pueden presentar un déficit temprano en la maduración del acceso vascular ${ }^{14,15}$. Existen diferentes factores preoperatorios asociados con una menor permeabilidad inmediata tras la creación de una FAVn:

- factores clínicos: mayores de 65 años, diabetes, sexo femenino, enfermedad arterial periférica y enfermedad coronaria;

- parámetros ecográficos: diámetro venoso inferior a $1.6 \mathrm{~mm}$, diámetro arterial inferior a 1.5 $\mathrm{mm}$, bajo flujo venoso y/o arterial y baja velocidad de flujo sanguíneo $0^{16-19}$.

En este contexto, resulta muy importante la identificación precoz de dicho déficit por parte del equipo de coordinación de la unidad vascular. El principal objetivo es aplicar todo un conjunto de medidas terapéuticas correctoras de tipo endovascular o quirúrgico encaminadas a evitar la colocación de catéteres venosos centrales para el inicio de hemodiálisis. Según los resultados obtenidos existe una buena concordancia 
entre la valoración clínica realizada por la enfermera de hemodiálisis de rol avanzado y el control ecográfico del nefrólogo (Índice Kappa $=0.539$ ). Tan sólo se detectó un caso de disconcordancia y por lo tanto la evaluación por parte de una enfermera especializada podría representar un primer paso en la detección de disfunciones precoces en el déficit de maduración.

A pesar del progresivo aumento en la edad media de la población incidente y prevalente en hemodiálisis nuestro grupo apuesta por la implantación de FAVn distales, con el principal objetivo de ahorrar capital venoso a nuestros pacientes. Nuestros resultados reportan los mayores efectos beneficiosos del ejercicio en este subgrupo de pacientes portadores de FAVn de localización distal (radio-cefálica). Estos resultados podrían deberse a efectos sistémicos secundarios a la realización del ejercicio en el acceso vascular. En cualquier caso, se trata de una intervención simple, con mínimos requerimientos de recursos, que puede facilitar y aumentar la permeabilidad y desarrollo de los accesos vasculares.

A pesar de que resultan necesarios la realización de estudios multicéntricos radomizados, podemos concluir a modo de resumen que un programa de ejercicio controlado post-quirúrgico favorece una adecuada maduración clínica, especialmente en el subgrupo de pacientes con FAVn distales. De acuerdo a nuestros resultados recomendamos la realización de una pauta controlada de ejercicio físico, bajo la supervisión de una enfermera experta en hemodiálisis de rol avanzado en el campo del acceso vascular.

Tabla 1. Descripción de la tabla de ejercicios en el grupo de estudio

Flexo-extensión de codo ( 2 ciclos de 10 repeticiones cada día): Sentado en una silla, pisar un extremo de la goma con el pie y el otro extremo cogerlo con la mano de la fístula. Doblar el codo hasta la máxima flexión y estirarlo lentamente, controlando la tensión de la goma. Vigilar la posición del hombro (que no se eleve) y la de la muñeca (mantenerla en posición neutra).

Flexo-extensión de muñeca ( 2 ciclos de 10 repeticiones cada día): Colocar el codo ligeramente flexionado y en supinación. Coger la goma con la mano y realizar la flexión palmar de la muñeca. Volver a una posición de salida lentamente para controlar la tensión de la goma. Es importante que el antebrazo no se mueva, sólo la muñeca. Si lo desea puede apoyar el antebrazo en una superficie y dejar la muñeca colgando.

Flexión máxima de dedos ( 2 ciclos de 25 repeticiones cada día): Poner la goma arrugada (hecha una bola) en la palma de la mano. Flexionar al máximo los dedos y presionar la goma. Soltar suavemente la presión.
Tabla 2. Características generales del grupo de estudio

\begin{tabular}{|l|l|}
\hline Variable & Media \pm DT \\
\hline Número pacientes & 69 pacientes \\
\hline Sexo & 48 varones / 21 mujeres \\
\hline Edad & $66.8 \pm 13.8$ años \\
\hline Pre-diálisis / Hemodiálisis & $44(65 \%) / 25(35 \%)$ \\
\hline FAVn distales / proximales & $29(42 \%) / 40(58 \%)$ \\
Hipertensión & $66(95.6 \%)$ \\
\hline Diabetes & $27(39.1 \%)$ \\
\hline Dislipemia & $26(37.6 \%)$ \\
Cardiopatía isquémica & $12(17.3 \%)$ \\
Enfermedad vascular periférica & $8(11.5 \%)$ \\
\hline
\end{tabular}

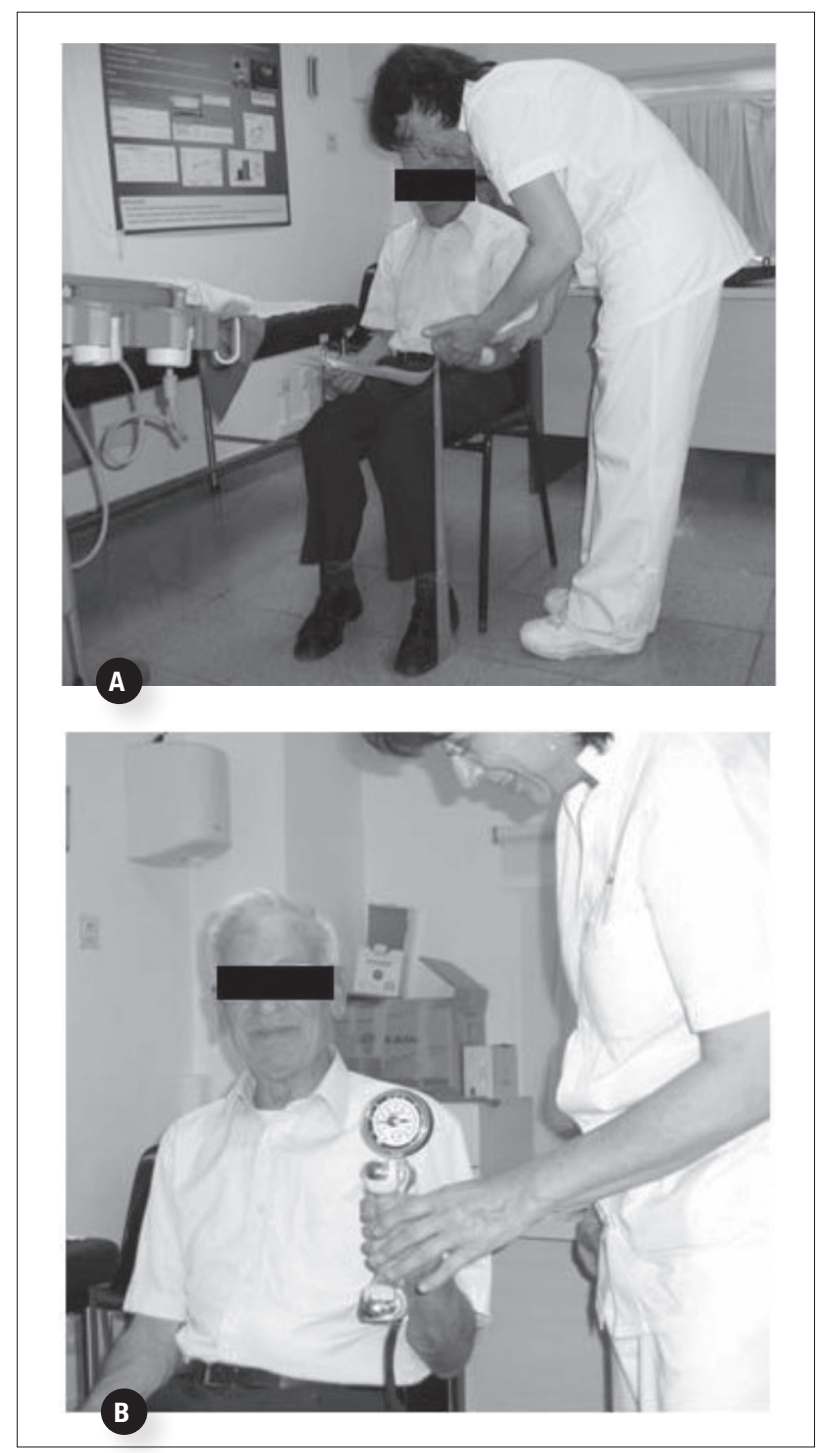

Figura 1. Explicación protocolizada de la pauta de ejercicios sobre la maduración de las FAVn. A.- Tabla de ejercicios con el tríptico explicativo. B.- Medida de la fuerza con el Hand-Dynamometer. 
A.- FAVn proximales

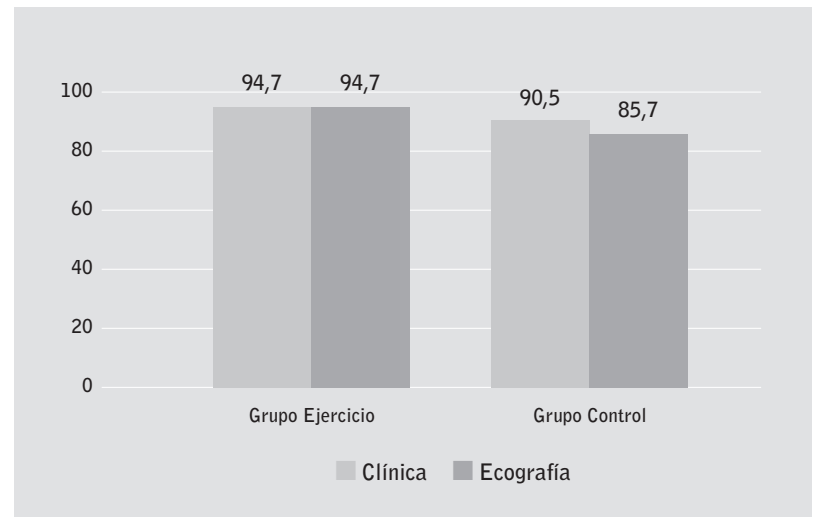

$94.7 \%$ vs $90.5 \%$ maduración clínica $(P=0.609)$ y $94.7 \%$ vs $85.7 \%$ ecográfica $(P=0.342)$.

\section{B.- FAVn distales}

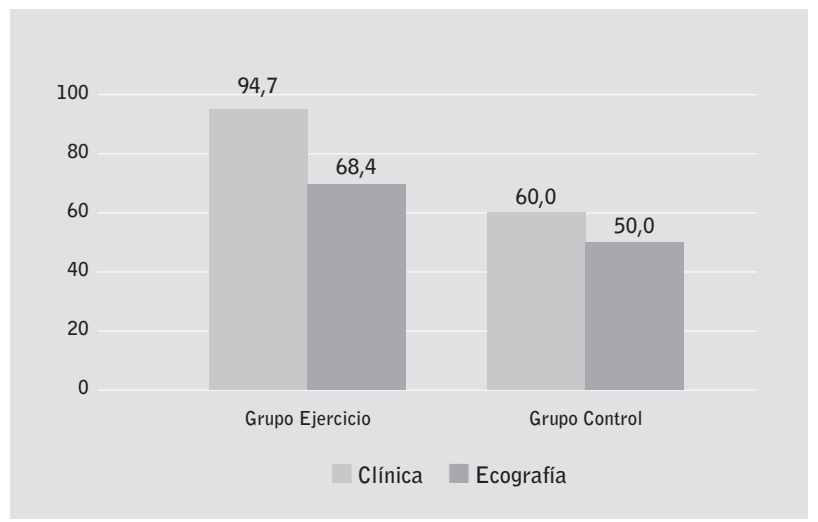

$94.7 \%$ vs $60 \%$ maduración clínica $(P=0.019)$ y $68.4 \%$ vs $50 \%$ ecográfica $(P=0.331)$.

Figura 2. Porcentaje de maduración clínica y ecográfica según la localización de la FAVn.

Recibido: 11 agosto 2015

Revisado: 16 agosto 2015

Modificado: 18 agosto 2015

Aceptado: 20 agosto 2015

\section{Bibliografía}

1. Gruss E, Portolés J, Caro P, Merino JL, LópezSánchez P, Tato A. et al. Los Modelos de atención al acceso vascular condicionan resultados heterogéneos en los centros de una misma comunidad. Nefrología 2010; 30: 310-6.

2. Roca Tey R. El acceso vascular para hemodiálisis: la asignatura pendiente. Nefrología 2010; 30: 280-7.

3. Lerma R, Callejas JM. Accesos vasculares para hemodiálisis: equipos multidisciplinarios. Angiología 2005; 57 (Supl 2): 169-76.

4. Fariñas MC, García-Palomo JD, Gutiérrez-Cuadra $M$. Infection associated with hemodialysis and peritoneal dialysis catheters Enferm Infecc Microbiol Clin 2008; 26: 518-26.

5. Allon M, Daurigas J, Depner TA, Greene T, Ornt D, Schwab SJ. Effect of change in vascular Access on patient mortality in hemodialysis patients. Am Kidney Dis 2006; 47: 469-77.

6. Department of Public Health: Catalonia registry. Statistical report 2013. http:// trasplantaments.gencat.cat/es/professionals/ registres_d_activitat_i_seguiment/registre_de malalts_renals/\#FW_bloc_5b8d0c8f-f18d-11e3997a005056339af7_4

7. Rodríguez JA, González E, Gutiérrez JM, et al. Guías de Acceso Vascular en Hemodialisis. Nefrología 2005; Volumen XXV (Supl 1): 1-07.

8. Tordoir J, Canaud B, Konner $\mathrm{K}$ et al. EBPG on vascular Access. Nephrol Dial Transplant 2007; 22 (Suppl 2): 88-117.

9. National Kidney Foundation. KDOQI Clinical Practice Guidelines and Clinical Practice Recomendations for 2006 Updates: Hemodialysis Adequacy, Peritoneal Dialysis Adequacy and Vascular Access. Am Kidney Dis 2006; 48 (Supl. 1) 1-322.

10. Leaf DA, MacRae HS-H, Grant E, Kraut J: Isometric exercise increases the size of forearm veins in patients with chronic renal failure. AmJ Med Sci 2003; 325: 115-119. 
11. Oder TF, Teodorescu V, Uribarri J: Effect of exercise on the diameter of arteriovenous fistulae in hemodialysis patients. ASAIO J 2003; 49: 554-555.

12. Uy $A L$, Jindal RM, Herndon TW, Yuan $C M$, Abbott $\mathrm{KC}$, Hurst FP. Impact of isometric handgrip exercises on cephalic vein diameter in non-AVF candidates, a pilot study. J Vasc Access 2013; 14:157-63.

13. Basile C, Casucci F, Lomonte C: Timing of first cannulation of arteriovenous fistula: time matters, but there is also something else. Nephrol Dial Transplant 2005; 20: 1519-1520.

14. Allon $M$, Robin $M L$. Increasing arteriovenous fistulas in hemodialysis patients: problems and solutions. Kidney Int 2002; 62:1109-1124.

15. Bradbury BD, Fissell RB, Albert JM, Anthony MS, Critchlow CW, Pisoni RL, et al. Predictors of early mortality among incident US hemodialysis patients in the Dialysis Outcomes and Practice Patterns Study (DOPPS). Clin J Am Soc Nephrol 2007; 2: 89-99.
16. Mestres G, Fontseré N, Campelos $P$, Maduell F, Riambau $V$. Intra-operative factors predicting 1-month arteriovenous fistula thrombosis. J Vasc Access 2012; 13:193-197.

17. Wong V, Ward R, Taylor J, Selvakumar S, How TV, Bakran A. Factors associated with early failure of arteriovenous fistulae for haemodialysis access. Eur J Vasc Endovas Surg 1996; 12: 207213.

18. Tordoir JH, Rooyens $P$, Dammers $R$, Van der Sande FM, De Haan M, Yo TI. Prospective evaluation of failure modes in autogenous radiocephalic wrist access for haemodialysis. Nephrol Dial Transplant 2003; 18: 378-83.

19. Lin $\mathrm{SL}$, Huang $\mathrm{CH}$, Chen HS, Hsu WA, Yen CJ, Yen TS. Effect of age and diabetes on blood flow rate and primary outcome of newly created hemodialysis arteriovenous fistulas. Am J Nephrol 1998; 18: 96100. 